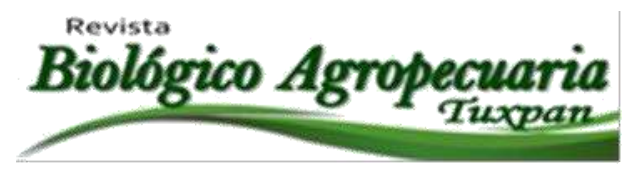

\title{
Diseño de una Granja Productora de Trucha Arcoiris en Guachochi, Chihuahua
}

\author{
A farm Design to Produce rainbow Trout in Guachochi, Chihuahua
}

\author{
Villalobos Gracia Irma Arisbel $^{1}$, Magaña Magaña José Eduardo ${ }^{2 凶}$, Villarreal Ramírez \\ Víctor Hugo $^{2}$, Hernández Salas José Esteban ${ }^{2}$, Núñez López Juan Javier ${ }^{2}$ \\ ${ }^{1}$ Estudiante de la maestría en Agronegocios, PNPC-CONACYT, ${ }^{2}$ Universidad \\ Autónoma de Chihuahua. Campus Delicias. Km 2.5 carretera Delicias-Rosales. CP. 33000. \\ Apartado postal 253. Delicias. Chihuahua. México \\ ${ }^{凶}$ Autor para correspondencia: emagana@uach.mx,
}

Recibido: $18 / 02 / 2017$

Aceptado: 30/06/2017

\section{RESUMEN}

Se evaluó la viabilidad de un proyecto que consiste en el establecimiento de una granja acuícola dedicada a la cría y venta de trucha arcoíris con el fin de aprovechar el clima y calidad del agua proveniente de un manantial, que son óptimos para la especie. El proyecto busca impactar en la creación de empleos estables en la región y derivar en un beneficio para sus propietarios. Se realizó una investigación de mercado que se basó en la aplicación de un cuestionario aleatorio de 190 encuestas en la comunidad de Guachochi, Chihuahua. Para codificar los datos se utilizó el programa SPSS versión 19. Los principales resultados indicaron que un 91\% consumen trucha arcoiris y un $99.5 \%$, están dispuestas a comprarla. Con base a este resultado se elaboró un proyecto de inversión el cual arrojo resultados positivos con una VAN $(15 \%)=\$ 51,491.76$, una TIR del $20 \%$ y una relación Beneficio/Costo $(15 \%)=1.081$, lo cual significa que el proyecto es viable. El impacto ambiental será mitigado mediante el uso de un cedazo para eliminar el exceso de materia orgánica para luego usar el agua para riego. Para desechar los peces muertos de la producción se contará con un lugar adecuado para su enterramiento. El proyecto no modificará la dinámica natural de ningún cuerpo de agua. El suelo no se verá afectado, ya que no se expondrá a materiales corrosivos. Se reducirá el impacto del consumo de energía eléctrica mediante el uso de paneles solares para el suministro de energía de la granja.

Palabras clave: Acuicultura, energía solar, producción en estanques, Sierra Tarahumara, trucha arcoiris

\section{ABSTRACT}

Was evaluated the feasibility of a project consisting in the establishment of an aquaculture farm dedicated to the breeding and sale of rainbow trout in order to take advantage of the climate and 
quality of the water from a spring, which is optimal for the species. The project aims to have an impact on the creation of stable jobs in the region and lead to a profit for its owners. He was a market research based on the application of a random questionnaire of 190 surveys in the community of Guachochi, Chihuahua. The program SPSS version 19 was used to encode the data. Main results indicated that $91 \%$ consume rainbow trout and a $99.5 \%$, are willing to buy it. Based on this result was elaborated an investment project which threw positive results with a $\operatorname{VAN}(15 \%)=\$ 51,491.76$, an IRR of $20 \%$ and a benefit/cost ratio $(15 \%)=1,081$, which means that the project is viable. The environmental impact will be mitigated by the using a sieve to remove excess organic matter to then use the water for irrigation. To discard the fish dead production will be the right place for his burial. The project will not change the natural dynamics of any body of water. The ground will not be affected, since it will not expose to corrosive materials. Will reduce the impact of the consumption of electrical energy through the use of solar panels to power the farm.

Key words: Aquaculture, solar energy, production in ponds, Sierra Tarahumara, rainbow trout.

\section{INTRODUCCIÓN}

De acuerdo a la FAO (2015), acuicultura es el cultivo de organismos acuáticos tanto en zonas costeras como del interior que implica intervenciones en el proceso de cría para aumentar la producción. Hoy en día es probablemente el sector de producción de alimentos de más rápido crecimiento y representa ahora casi el $50 \%$ del pescado destinado a la alimentación a nivel mundial. Cuando se utilizan las especies y técnicas adecuadas puede compararse de manera ventajosa con la avicultura, porcicultura, y la cría de bovinos con respecto a sus rendimientos económicos. Mejora el ingreso de las zonas rurales, esto particularmente en los países en desarrollo. Otra de las ventajas que se tienen de poner en práctica la acuicultura, según Huet (1998), es que permite darle uso a terrenos que de otra forma seguirían improductivos, lo cual es de gran ayuda sobre todo en las regiones de clima templado.

La trucha arcoíris es un salmónido originario de América del Norte. Es un pez cuyo hábitat son las aguas frías, vive en corrientes de agua, estanques, lagos y también es la más apropiada para la producción para consumo, ya que se adapta mejor que otras especies, se presta más a la domesticación y a la alimentación artificial, soporta más las temperaturas elevadas y el menor oxígeno. Según Panne y Luchini (2012), esta especie puede llegar a soportar incluso hasta $20^{\circ} \mathrm{C}$, aunque su temperatura ideal son $15^{\circ} \mathrm{C}$. Su carne es un alimento de gran calidad nutricional para el ser humano, ya que su contenido en proteína es excelente, la textura de su carne facilita su asimilación y es ideal como complemento alimenticio, ya que $250 \mathrm{~g}$ de su carne aportan el $88 \%$ de las proteínas requeridas por un niño y el $68 \%$ de lo que requiere un adolescente (SAGARPA (2012).

Los principales productores de trucha arcoíris en el mundo son: Europa, Norteamérica, Chile, Japón y Australia, mientras que en nuestro país, las entidades que se dedican principalmente al cultivo de la trucha arcoíris son: Sonora, Chihuahua, Jalisco, Durango, Veracruz, Puebla, Hidalgo, Tlaxcala, Michoacán, Guanajuato, Guerrero, Querétaro, Estado de México y Oaxaca. En el Estado de Chihuahua los 
principales municipios que la producen son Madera, Guachochi, Guadalupe y Calvo y Bocoyna.

Con el fin de aprovechar la oportunidad de negocio que existe debido a las condiciones del clima y calidad del agua adecuadas para el cultivo de la trucha, y, con la intención de que dicho proyecto origine empleos estables en la región y derive en un beneficio económico para los propietarios, se evaluó la oportunidad de poner en marcha un proyecto que consiste en el establecimiento de una granja acuícola dedicada a la cría, engorda y venta de trucha arcoíris, en el municipio de Guachochi, Chihuahua.

\section{Objetivos:}

\section{Objetivo general.}

Contribuir al desarrollo económico del municipio de Guachochi, Chihuahua a través del diseño de una granja productora de trucha arcoíris en estanques.

\section{Objetivos específicos.}

1. Elaborar el proyecto de inversión de una granja productora de trucha arcoíris en estanques en el municipio de Guachochi, Chihuahua y alcanzar una producción de 10.8 toneladas por año para 2018.

2. Someter el proyecto de inversión a una convocatoria para conseguir recursos para su operación.

\section{MATERIALES Y MÉTODOS}

\section{Localización y descripción especifica del sitio del proyecto:}

El proyecto se elaboró en el municipio de Guachochi, Chihuahua:

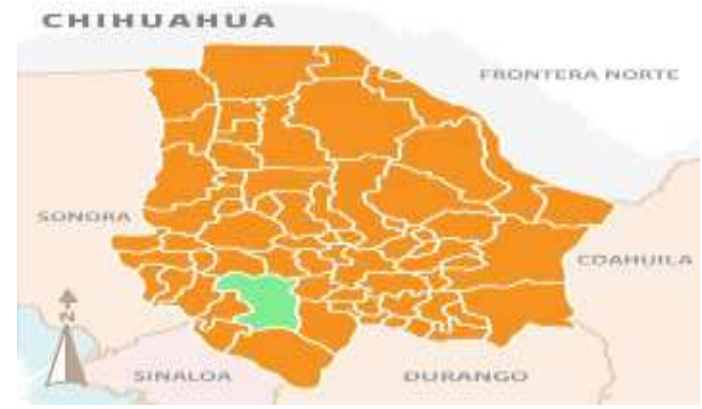

Figura 1: Localización del municipio de Guachochi, Chihuahua.

El municipio de Guachochi, Chihuahua está ubicado en la latitud Norte, 2649', longitud oeste $107^{\circ} 04^{\prime}$ 'y a una altitud de 2400 metros sobre el nivel del mar. Tiene una extensión territorial de 4,340. $35 \mathrm{~km}^{2}$. Guachochi cuenta con una población total de 45,881 habitantes, de los cuales un $70 \%$ son de etnia rarámuri.

Microlocalización del área del proyecto. Se eligió el rancho "El Lobito", comunidad de Guachochi, Chihuahua para la construcción de la granja, ya que el señor Eusebio Acosta Moreno, miembro del grupo, cuenta con el terreno necesario y con un manantial que es la fuente ideal de agua limpia y clara, vital para la cría de la trucha arcoiris.

Organización. Se reunieron los miembros del grupo y se llevó a cabo un taller de planeación estratégica participativa, en la cual se obtuvieron las fortalezas, debilidades, oportunidades y amenazas del proyecto, así como también se diseñaron la misión, visión y valores de la empresa. El grupo decidió que de aprobarse el apoyo por parte de la SAGARPA se creara una persona moral como Sociedad de Producción Rural.

Razón Social. El nombre de la granja será: "Grupo Las Truchas". 


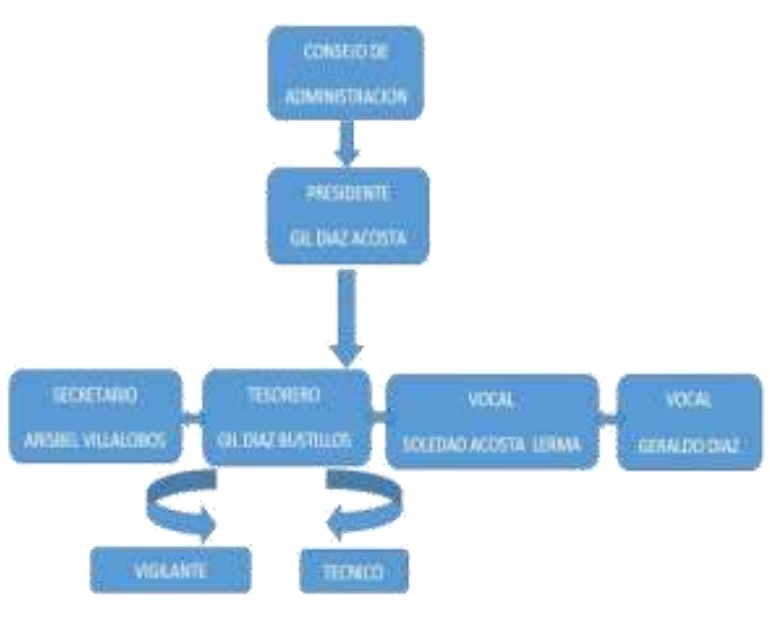

Figura 2. Organigrama del Consejo de Administración.

Descripción y análisis de materias primas, productos y subproductos. Se producirá trucha arcoíris ya que se dan las condiciones favorables para su cría y aprovechamiento.

Materias primas. Para la producción de la trucha arcoíris, los insumos necesarios son alevines, alimento para alevines en sus diferentes estadíos y medicinas de ser necesarias por alguna enfermedad.

Precio. El precio varía según las condiciones del lugar de cría y de la alimentación, fluctuando entre $\$ 80.00$ y $\$ 100.00$ el kilogramo.

Clientes. La viabilidad de compra de la trucha arcoíris se basa en su sabor, carne suave y en que es un excelente complemento alimenticio para toda la familia, especialmente para niños y adolescentes.

El producto se venderá directamente en la granja a los habitantes de la región y también se transportara en camioneta a los pueblos vecinos para distribuirla en los comercios y restaurantes con los que se tendrá previo acuerdo.
Análisis del mercado. Se aplicó un sondeo exploratorio de 15 encuestas basado en el consumo familiar de pescado en la comunidad de Guachochi y, en base a sus resultados se determinó el tamaño de muestra por medio del método de intervalo de confianza (Ver figura 1). Enseguida se aplicó el cuestionario a 190 habitantes de la comunidad con el fin de sondear el consumo y demanda de trucha arcoiris. Para la codificar los datos se utilizó el programa estadístico SPSS (Statistics Package for the Social Sciences) versión 19.

Análisis de la fuente de agua para la instalación de la granja. Se tomó muestra del manantial que será la fuente de agua para la construcción de la granja y se analizaron las mismas en el laboratorio de análisis de suelo, agua y plantas de la Facultad de

Ciencias Agrícolas y Forestales, concluyéndose que el agua es apta para la sanidad e inocuidad de los peces, ya que se encuentra entre los parámetros requeridos por la especie. (Ver figuras 1 y 2: análisis de la fuente de agua).

Proyecto de inversión. Toda vez que conozcamos los resultados del estudio de mercado y el análisis del agua, se elaborara un proyecto de inversión, el cual considera el análisis organizacional de la empresa, el estudio de mercado, ingeniería del proyecto, costos de producción, fuentes de financiamiento, análisis financiero e impactos ambientales y económicos. Se considera utilizar el formato de FAPPASAGARPA, 2016.

\section{RESULTADOS Y DISCUSIONES}

Misión: "Somos una empresa familiar dedicada a la producción y comercialización de trucha arcoíris; ofreciendo un producto fresco, saludable y de calidad, trabajando en equipo para satisfacer las necesidades de 
nuestros clientes y así generar empleo y desarrollo en el municipio de Guachochi, Chihuahua".

Visión: Consolidarnos como una empresa competitiva en la producción y comercialización de trucha arcoíris de manera sustentable, en el año 2018; no solo vendiendo nuestro producto localmente sino expandiendo nuestras ventas a los municipios vecinos. También aumentar gradualmente nuestra producción después de la primera cosecha.

Valores: Higiene, honestidad, responsabilidad, atención al cliente, disciplina.

Estudio de mercado. Se llevó a cabo la elaboración y aplicación del cuestionario piloto por medio del método de intervalo de confianza y en base a sus resultados se determinó el tamaño de muestra por medio del método de intervalo de confianza:

$$
\mathbf{Z}^{2}\left(\boldsymbol{\sigma}^{2}\right)
$$

$\mathbf{n}=$

\section{$\mathbf{D}^{2}$}

Dónde:

$\mathbf{Z}=95 \%=1.96$

$\mathbf{D}=$ Del 3 al 10\% de la media

Media: $2.87(5 \%)=0.1485$

Varianza: .981

$$
\mathrm{n}=\frac{(1.96)^{2}(0.981)}{190(0.1435)^{2}}=183.03=
$$

Enseguida se aplicó el cuestionario por medio de muestreo aleatorio simple a 190 habitantes del municipio de Guachochi, Chihuahua, obteniéndose los siguientes resultados:

\begin{tabular}{|c|c|c|c|c|c|c|}
\hline \multirow{2}{*}{$\begin{array}{c}\text { Que tipo de } \\
\text { pescado consume }\end{array}$} & \multicolumn{5}{|c|}{ ¿Cuanto pescado consume usted y su familia por mes? } & \multirow[b]{2}{*}{ Total } \\
\hline & $1.1 \mathrm{a} 3 \mathrm{kgr}$ & $3.1 \mathrm{a} 5 \mathrm{kgr}$ & $5.1 \mathrm{a} 8 \mathrm{kgr}$ & 8,1 a $10 \mathrm{kgr}$ & $\begin{array}{c}\text { mayor de } \\
10 \mathrm{kgr}\end{array}$ & \\
\hline \multirow{4}{*}{ Tilapia } & 1 & 2 & 0 & 0 & 0 & 3 \\
\hline & $33 \%$ & $67 \%$ & $0 \%$ & $0 \%$ & $0 \%$ & $100 \%$ \\
\hline & $1 \%$ & $5 \%$ & $0 \%$ & $0 \%$ & $0 \%$ & $2 \%$ \\
\hline & $1 \%$ & $1 \%$ & $0 \%$ & $0 \%$ & $0 \%$ & $2 \%$ \\
\hline \multirow{4}{*}{ Trucha arcoiris } & 74 & 27 & 13 & 9 & 9 & 132 \\
\hline & $56 \%$ & $20 \%$ & $10 \%$ & $7 \%$ & $7 \%$ & $100 \%$ \\
\hline & $75 \%$ & $61 \%$ & $65 \%$ & $69 \%$ & $64 \%$ & $69 \%$ \\
\hline & $39 \%$ & $14 \%$ & $7 \%$ & $5 \%$ & $5 \%$ & $69 \%$ \\
\hline \multirow{4}{*}{ Black bass } & 0 & 2 & 2 & 0 & 1 & 5 \\
\hline & $0 \%$ & $40 \%$ & $40 \%$ & $0 \%$ & $20 \%$ & $100 \%$ \\
\hline & $0 \%$ & $5 \%$ & $10 \%$ & $0 \%$ & $7 \%$ & $3 \%$ \\
\hline & $0 \%$ & $1 \%$ & $1 \%$ & $0 \%$ & $1 \%$ & $3 \%$ \\
\hline \multirow{4}{*}{ carpa } & 3 & 2 & 2 & 1 & 0 & 8 \\
\hline & $38 \%$ & $25 \%$ & $25 \%$ & $13 \%$ & $0 \%$ & $100 \%$ \\
\hline & $3 \%$ & $5 \%$ & $10 \%$ & $8 \%$ & $0 \%$ & $4 \%$ \\
\hline & $2 \%$ & $1 \%$ & $1 \%$ & $1 \%$ & $0 \%$ & $4 \%$ \\
\hline \multirow{4}{*}{$\begin{array}{c}\text { Trucha arcoiris, black } \\
\text { bass }\end{array}$} & 3 & 3 & 1 & 2 & 2 & 11 \\
\hline & $27 \%$ & $27 \%$ & $9 \%$ & $18 \%$ & $18 \%$ & $100 \%$ \\
\hline & $3 \%$ & $7 \%$ & $5 \%$ & $15 \%$ & $14 \%$ & $6 \%$ \\
\hline & $2 \%$ & $2 \%$ & $1 \%$ & $1 \%$ & $1 \%$ & $6 \%$ \\
\hline \multirow{4}{*}{$\begin{array}{l}\text { Tilapia, trucha } \\
\text { arcoiris }\end{array}$} & 6 & 2 & 0 & 0 & 1 & 9 \\
\hline & $67 \%$ & $22 \%$ & $0 \%$ & $0 \%$ & $11 \%$ & $100 \%$ \\
\hline & $6 \%$ & $5 \%$ & $0 \%$ & $0 \%$ & $7 \%$ & $5 \%$ \\
\hline & $3 \%$ & $1 \%$ & $0 \%$ & $0 \%$ & $1 \%$ & $5 \%$ \\
\hline \multirow{4}{*}{ Trucha arcoiris, bagre } & 4 & 1 & 0 & 0 & 0 & 5 \\
\hline & $80 \%$ & $20 \%$ & $0 \%$ & $0 \%$ & $0 \%$ & $100 \%$ \\
\hline & $4 \%$ & $2 \%$ & $0 \%$ & $0 \%$ & $0 \%$ & $3 \%$ \\
\hline & $2 \%$ & $1 \%$ & $0 \%$ & $0 \%$ & $0 \%$ & $3 \%$ \\
\hline \multirow{4}{*}{$\begin{array}{c}\text { Tilapia, trucha } \\
\text { arcoiris, black bass }\end{array}$} & 1 & 1 & 0 & 0 & 0 & 2 \\
\hline & $50 \%$ & $50 \%$ & $0 \%$ & $0 \%$ & $0 \%$ & $100 \%$ \\
\hline & $1 \%$ & $2 \%$ & $0 \%$ & $0 \%$ & $0 \%$ & $1 \%$ \\
\hline & $1 \%$ & $1 \%$ & $0 \%$ & $0 \%$ & $0 \%$ & $1 \%$ \\
\hline \multirow{4}{*}{$\begin{array}{c}\text { Tilapia, trucha } \\
\text { arcoiris, black bass, } \\
\text { carpa }\end{array}$} & 0 & 0 & 0 & 0 & 1 & 1 \\
\hline & $0 \%$ & $0 \%$ & $0 \%$ & $0 \%$ & $100 \%$ & $100 \%$ \\
\hline & $0 \%$ & $0 \%$ & $0 \%$ & $0 \%$ & $7 \%$ & $1 \%$ \\
\hline & $0 \%$ & $0 \%$ & $0 \%$ & $0 \%$ & $1 \%$ & $1 \%$ \\
\hline \multirow{4}{*}{ Trucha arcoiris, carpa } & 4 & 0 & 2 & 1 & 0 & 7 \\
\hline & $57 \%$ & $0 \%$ & $29 \%$ & $14 \%$ & $0 \%$ & $100 \%$ \\
\hline & $4 \%$ & $0 \%$ & $10 \%$ & $8 \%$ & $0 \%$ & $4 \%$ \\
\hline & $2 \%$ & $0 \%$ & $1 \%$ & $1 \%$ & $0 \%$ & $4 \%$ \\
\hline
\end{tabular}

Cuadro 1. ¿Qué tipo de pescado consume?/ ¿Cuánto pescado consume usted y su familia por mes? 


\begin{tabular}{|c|c|c|c|c|c|c|}
\hline \multirow{2}{*}{$\begin{array}{c}\text { Que tipo de } \\
\text { pescado consume }\end{array}$} & \multicolumn{5}{|c|}{ ¿Cuanto pescado consume usted y su familia por mes? } & \multirow[b]{2}{*}{ Tota } \\
\hline & $1.1 \mathrm{a} 3 \mathrm{kgr}$ & 3.1 a $5 \mathrm{kgr}$ & $5.1 \mathrm{a} 8 \mathrm{kgr}$ & 8,1 a $10 \mathrm{kgr}$ & $\begin{array}{c}\text { mayor de } \\
10 \mathrm{kgr}\end{array}$ & \\
\hline \multirow{4}{*}{$\begin{array}{l}\text { Tilapia, trucha } \\
\text { arcoiris, carpa }\end{array}$} & 1 & 2 & 0 & 0 & 0 & 3 \\
\hline & $33 \%$ & $67 \%$ & $0 \%$ & $0 \%$ & $0 \%$ & $100 \%$ \\
\hline & $1 \%$ & $5 \%$ & $0 \%$ & $0 \%$ & $0 \%$ & $2 \%$ \\
\hline & $1 \%$ & $1 \%$ & $0 \%$ & $0 \%$ & $0 \%$ & $2 \%$ \\
\hline \multirow{4}{*}{ Bagre, carpa } & 1 & 0 & 0 & 0 & 0 & 1 \\
\hline & $100 \%$ & $0 \%$ & $0 \%$ & $0 \%$ & $0 \%$ & $100 \%$ \\
\hline & $1 \%$ & $0 \%$ & $0 \%$ & $0 \%$ & $0 \%$ & $1 \%$ \\
\hline & $1 \%$ & $0 \%$ & $0 \%$ & $0 \%$ & $0 \%$ & $1 \%$ \\
\hline \multirow{4}{*}{$\begin{array}{c}\text { Tilapia, trucha } \\
\text { arcoiris, black bass, } \\
\text { bagre, carpa }\end{array}$} & 0 & 2 & 0 & 0 & 0 & 2 \\
\hline & $0 \%$ & $100 \%$ & $0 \%$ & $0 \%$ & $0 \%$ & $100 \%$ \\
\hline & $0 \%$ & $5 \%$ & $0 \%$ & $0 \%$ & $0 \%$ & $1 \%$ \\
\hline & $0 \%$ & $1 \%$ & $0 \%$ & $0 \%$ & $0 \%$ & $1 \%$ \\
\hline \multirow{4}{*}{$\begin{array}{c}\text { Trucha arcoiris, } \\
\text { bagre, carpa }\end{array}$} & 1 & 0 & 0 & 0 & 0 & 1 \\
\hline & $100 \%$ & $0 \%$ & $0 \%$ & $0 \%$ & $0 \%$ & $100 \%$ \\
\hline & $1 \%$ & $0 \%$ & $0 \%$ & $0 \%$ & $0 \%$ & $1 \%$ \\
\hline & $1 \%$ & $0 \%$ & $0 \%$ & $0 \%$ & $0 \%$ & $1 \%$ \\
\hline \multirow{4}{*}{ Total } & 99 & 44 & 20 & 13 & 14 & 190 \\
\hline & $52 \%$ & $23 \%$ & $11 \%$ & $7 \%$ & $7 \%$ & $100 \%$ \\
\hline & $100 \%$ & $100 \%$ & $100 \%$ & $100 \%$ & $100 \%$ & $100 \%$ \\
\hline & $52 \%$ & $23 \%$ & $11 \%$ & $7 \%$ & $7 \%$ & $100 \%$ \\
\hline
\end{tabular}

En el cuadro número uno se observa que las personas que quieren comprar exclusivamente trucha arcoiris representan el $69.5 \%$ del total (132 personas). De las cuales $56.1 \%$ (74 personas), consumen de 1.1 a $3 \mathrm{~kg}$ por mes; $20.5 \%$ (27 personas), consumen de 3.1 a $5 \mathrm{~kg}$ por mes; el $9.8 \%$ (13 personas), consumen de 5.1 a $8 \mathrm{~kg}$ por mes; el $6.8 \%$ (9 personas), consumen de 8.1 a $10 \mathrm{~kg}$ y el $6.8 \%$ (9 personas), consumen más de $10 \mathrm{~kg}$ por mes.

Tenemos como resultado que 173 personas consumen trucha arcoíris; esto es: $91.1 \%$ del total de la población encuestada consume trucha arcoiris en tanto que el $8.9 \%$ restante (17 personas) prefiere otra variedad de pescado.
Grafica 1. ¿Compra el pescado fresco o congelado?/ ¿Cuánto pescado consume usted y su familia por mes?

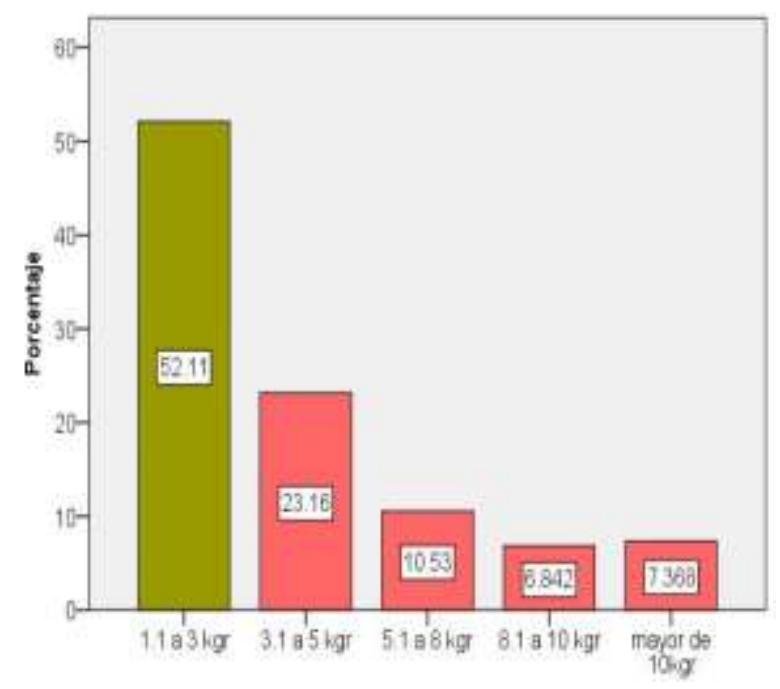

En la gráfica 1 se observa que el $52.11 \%$ del total de las personas entrevistadas consumen de 1.1 a $3 \mathrm{~kg}$, el $23.16 \%$ de 3.1 a $5 \mathrm{~kg}$. Esto nos indica la preferencia del pescado en la alimentación de la región estudiada. 
Cuadro 3. ¿Qué tipo de pescado consume?/¿Compra el pescado fresco congelado?

\begin{tabular}{|c|c|c|c|c|c|c|c|}
\hline \multirow{2}{*}{$\begin{array}{c}\text { Tipo de } \\
\text { pescado }\end{array}$} & Fresco & Congelado & $\begin{array}{c}\text { Fresco, } \\
\text { Congelado }\end{array}$ & Total & $\begin{array}{l}\% \\
\text { Fresco }\end{array}$ & $\begin{array}{c}\% \\
\text { Congela } \\
\text { do }\end{array}$ & $\begin{array}{c}\% \text { fresco, } \\
\text { Congelado }\end{array}$ \\
\hline Tilapia & 1 & 2 & 0 & 3 & $33.3 \%$ & $66.7 \%$ & $0 \%$ \\
\hline Black Bass & 3 & 1 & 1 & 5 & $60.0 \%$ & $20.0 \%$ & $20.0 \%$ \\
\hline Carpa,bagre & 8 & 1 & 0 & 9 & $88.9 \%$ & $11.1 \%$ & $0 \%$ \\
\hline $\begin{array}{c}\text { Trucha } \\
\text { arcoiris }\end{array}$ & 147 & 21 & 5 & 173 & $85.0 \%$ & $12.1 \%$ & $2.9 \%$ \\
\hline TOTAL & 159 & 25 & 6 & 190 & $83.7 \%$ & $13.2 \%$ & $3.2 \%$ \\
\hline
\end{tabular}

En el cuadro número tres se observa que el $83.7 \%$ de las personas prefieren comprar pescado fresco, $13.2 \%$ congelado y $3.2 \%$ en ambas formas. En tanto que de las que consumen trucha arcoiris, el $85 \%$ prefiere comprarlas frescas, el $12.1 \%$ congeladas y el $2.9 \%$ fresca $y$ congelada

Cuadro 4. ¿Qué tipo de pescado consume?/¿Estaría interesado en adquirir trucha arcoiris fresca producida aquí en la región?

\begin{tabular}{|c|c|c|c|c|c|}
\hline \multirow{2}{*}{$\begin{array}{c}\text { ¿Qué tipo de pescado } \\
\text { consume? }\end{array}$} & \multicolumn{6}{|c|}{ ¿Estaría interesado en adquirir trucha arcoiris fresca producida aquí } \\
\cline { 2 - 6 } & $\mathrm{Si}$ & $\mathrm{No}$ & Total & $\%$ Si sobre el total & $\begin{array}{c}\text { \% No sobre el } \\
\text { total }\end{array}$ \\
\hline Tilapia & 3 & 0 & 3 & $1.6 \%$ & $0 \%$ \\
\hline Black Bass & 4 & 1 & 5 & $2.1 \%$ & $0.5 \%$ \\
\hline Carpa,bagre & 9 & 0 & 9 & $4.7 \%$ & $0 \%$ \\
\hline Trucha arcoiris & 173 & 0 & 173 & $91.1 \%$ & $0 \%$ \\
\hline TOTAL & 189 & 1 & 190 & $99.5 \%$ & $0.5 \%$ \\
\hline
\end{tabular}

En el cuadro cuatro se concluye que el 91.1\% (173 personas de las 190 de la muestra), consumen trucha arcoiris. Y esas 173 personas estarían dispuestas a adquirirla fresca y producida en la región.
También tenemos que del total (190 personas), un 99.5\%(189 personas), están dispuestas a adquirir pescado fresco producido en la región; y tan solo un $0.5 \%$ (1 persona), no estaría dispuesta a consumirlo. 
Ingeniería del proyecto: $\mathrm{El}$ proyecto constara de cuatro estanques de $30 \times 3 \times 1 \mathrm{~m}$ $\left(90 \mathrm{~m}^{3}\right)$ para la cría de la trucha en sus diferentes etapas, una pila de almacenamiento de $100 \mathrm{~m}^{3}$, la cual estará techada, una oficina, y un almacén. Cada estanque será independiente, se va a cambiar el agua cada segundo o tercer día, conforme se vaya necesitando.

Pila de $5 \times 10 \times 2=100 \mathrm{~m}^{3}$. Se escarbara para hacerla 2 metros para que quede con una pendiente y que baje el agua de manera natural del ojo de agua a la pila. Del ojo de agua a la pila habrá 2 metros de distancia. De la pila al primer estanque $10 \mathrm{~m}$ de distancia, de la pila al segundo estanque: 13 $\mathrm{m}$ distancia, de la pila al tercer estanque: 16 $\mathrm{m}$ y de la pila al cuarto estanque $19 \mathrm{~m}$. Entre estanque y estanque habrá $3 \mathrm{~m}$ de distancia. La profundidad de la bomba será de $2 \mathrm{~m}$ y los paneles solares estarán a un lado de la pila.

Luego de terminar el análisis del mercado se creó un grupo de socios integrado por seis personas con el fin de someter el proyecto a una convocatoria con el propósito de obtener recursos para la operación del proyecto.

Costos de producción $y$ fuentes de financiamiento. Se llevó a cabo una cotización de materiales de construcción y también del precio de los alevines, alimento y medicinas, mano de obra, y todos los gastos directos e indirectos para la construcción de la granja, dando como

resultado una inversión total de $\$ 499,490.11$. Después de llevar a cabo el presupuesto de inversión se calculó la depreciación de oficinas, almacén, pila de almacenamiento, los cuatro estanques, la bomba, los paneles y de toda la granja de acuerdo a su respectiva tasa anual (ver cuadro 5. Depreciaciones). Enseguida se sumaron todas las depreciaciones para calcular la proyección financiera (Ver cuadro 6. Proyección financiera a 5 años).

Se sometió el proyecto a la convocatoria de la Secretaria de Agricultura, Ganadería, Desarrollo Rural, Pesca y Alimentación (SAGARPA), mediante el Programa Fondo para el Apoyo a Proyectos Productivos en Núcleos Agrarios (FAPPA) 2016 para la obtención de recursos para su operación, solicitando la cantidad de $\$ 240,000.00$ a razón de $\$ 40,000.00$ por socio. $\mathrm{La}$ aportación de los socios sería de $\$ 211,545.90$ (46.85\%) y la de FAPPA sería del 53.15\% (\$240,000). (Ver figuras 3: mano de obra y 4: costo y alimentación de alevines).

\section{Análisis financiero:}

\section{Cuadro 5. Depreciaciones}

\begin{tabular}{|c|c|c|c|c|}
\hline \multicolumn{5}{|c|}{ DEPRECIACIONES } \\
\hline CONCEPTO & VALOR ORIGINAL & TASA & $\begin{array}{l}\text { DEPRECIACION } \\
\text { ANUAL }\end{array}$ & AÑOS \\
\hline Oficinas & $\$ 100,928.58$ & $3.30 \%$ & $\$ 3,364.29$ & 30 \\
\hline Almacen & $\$ 93,131.67$ & $5.00 \%$ & $\$ 4,656.58$ & 20 \\
\hline $\begin{array}{c}\text { Pila de } \\
\text { almacenamiento }\end{array}$ & $\$ 19,337.58$ & $4 \%$ & $\$ 773.50$ & 25 \\
\hline 4 Estanques & $\$ 214,627.68$ & $4 \%$ & $\$ 10,731.38$ & 20 \\
\hline $\begin{array}{l}\text { Bomba de } 1 \mathrm{HP} \\
\text { energia directa }\end{array}$ & $\$ 35,700.00$ & $10 \%$ & $\$ 3,570.00$ & 10 \\
\hline $\begin{array}{l}\text { Paneles solares } \\
250 \mathrm{~W} \text { (6 piezas) }\end{array}$ & $\$ 26,400.00$ & $10 \%$ & $\$ 2,640.00$ & 10 \\
\hline $\begin{array}{c}\text { Sistema de } \\
\text { cableado solar. }\end{array}$ & $\$ 3,536.20$ & $10 \%$ & $\$ 353.62$ & 10 \\
\hline $\begin{array}{c}\text { Tubo PVC } \\
\text { hidraulico de 2", } \\
\text { de } 6 \mathrm{~m} \text { (4 piezas). }\end{array}$ & $\$ 422.00$ & $10 \%$ & $\$ 42.20$ & 10 \\
\hline $\begin{array}{c}\text { Cople de PVC } \\
\text { hidraulico de 2"(4 } \\
\text { piezas) }\end{array}$ & $\$ 68.80$ & $10 \%$ & $\$ 6.88$ & 10 \\
\hline $\begin{array}{l}\text { Tapadera de } 2 " \text { de } \\
\text { PVC hidraulico ( } 2 \\
\text { piezas). }\end{array}$ & $\$ 27.00$ & $10 \%$ & $\$ 2.70$ & 10 \\
\hline $\begin{array}{c}\text { Lamina } \\
\text { galvanizada de } \\
\text { 20FT (10 piezas) }\end{array}$ & $\$ 3,570.00$ & $10 \%$ & $\$ 357.00$ & 10 \\
\hline $\begin{array}{c}\text { Polin de 6X6 (6 } \\
\text { piezas). }\end{array}$ & $\$ 1,491.60$ & $10 \%$ & $\$ 149.16$ & 10 \\
\hline $\begin{array}{l}\text { Pija lamina (300 } \\
\text { piezas). }\end{array}$ & $\$ 0.83$ & $10 \%$ & $\$ 0.08$ & 10 \\
\hline & TOTAL & & $\$ 26,647.40$ & \\
\hline
\end{tabular}




\section{Cuadro 6. Proyección financiera a 5 años}

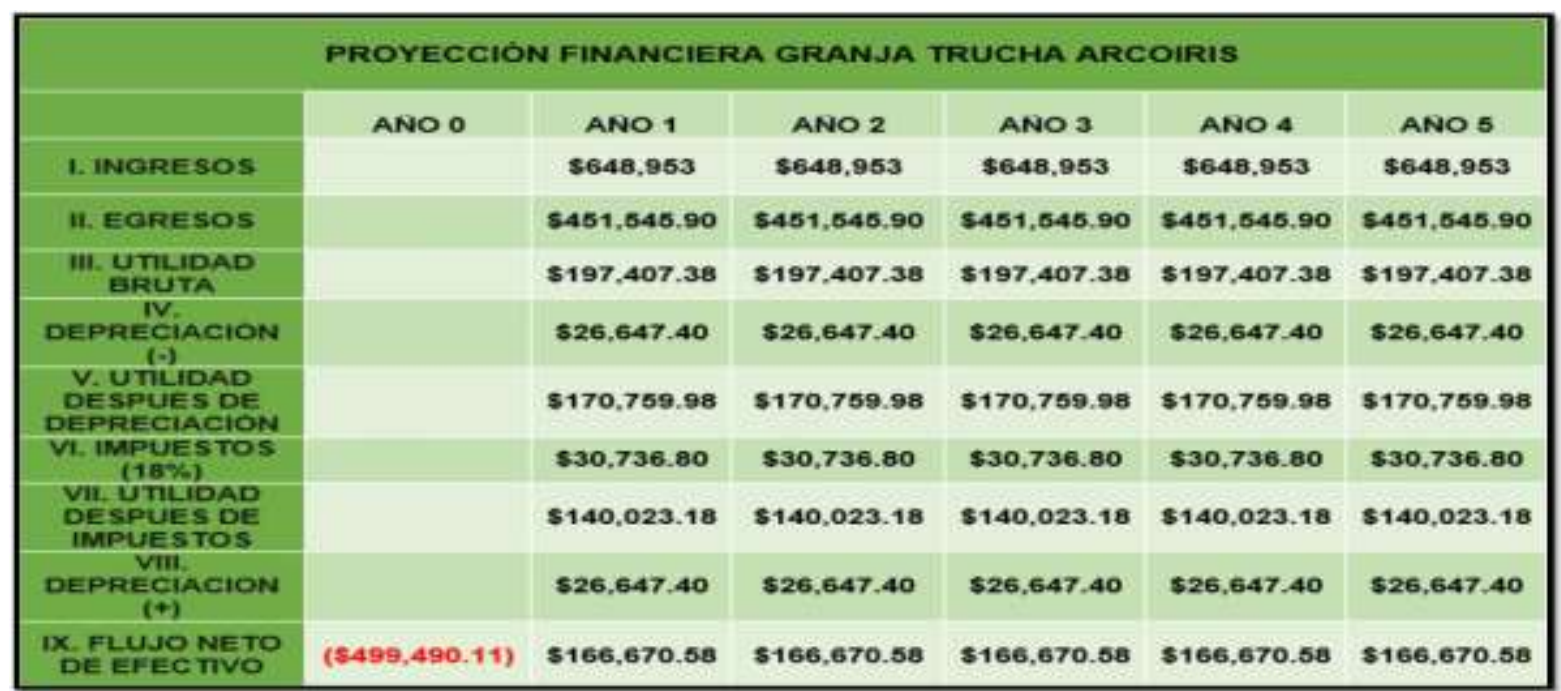

El proyecto de inversión presenta indicadores de rentabilidad positivos, lo que significa que es viable y se puede invertir en él, como podemos observar en el (cuadro 7. Indicadores de rentabilidad).

\section{Cuadro 7. Indicadores de rentabilidad}

\begin{tabular}{|c|c|c|l|}
\hline $\begin{array}{c}\text { Indicador de } \\
\text { rentabilidad }\end{array}$ & $\begin{array}{c}\text { Porcentaje/ } \\
\text { Cantidad }\end{array}$ & Resultado & \multicolumn{1}{c|}{ Interpretación } \\
\hline $\begin{array}{c}\text { Tasa interna } \\
\text { de retorno } \\
\text { (TIR) }\end{array}$ & $20 \%$ & Se acepta & $\begin{array}{l}\text { El criterio de aceptación para proyectos de desarrollo } \\
\text { social es el de aceptar el proyecto que tenga una TIR } \\
\text { ligeramente mayor que el costo de oportunidad del } \\
\text { capital vigente en el mercado. En este caso dicho } \\
\text { costo de oportunidad fue del 15\%. Bajo esta condición } \\
\text { el presente proyecto es viable. }\end{array}$ \\
\hline $\begin{array}{c}\text { Valor actual } \\
\text { neto (VAN) }\end{array}$ & $15 \%$ & $\$ 51,491.7$ & $\begin{array}{l}\text { El Valor Actual Neto (VAN) es el valor actual de los } \\
\text { flujos futuros de efectivo descontados a una tasa del } \\
15 \% \text {.La VAN del proyecto de inversión analizado es } \\
\text { positivo \$51,491.76 (mayor o igual a 0). Bajo este } \\
\text { criterio el proyecto es viable. }\end{array}$ \\
\hline $\begin{array}{c}\text { Relación } \\
\text { costo/benefici } \\
\text { o (REL B/C) }\end{array}$ & $15 \%$ & 1.081 & $\begin{array}{l}\text { Toma lo ingresos y egresos de la proyección } \\
\text { financiera para determinar cuáles son los beneficios } \\
\text { acepta el proyecto cuya relación beneficio /costo sea } \\
\text { igual o mayor a 1. Bajo este criterio se acepta el } \\
\text { proyecto. }\end{array}$ \\
\hline
\end{tabular}


Por último se sometió el proyecto a la convocatoria de la Secretaria de Agricultura, Ganadería, Desarrollo Rural, Pesca y Alimentación (SAGARPA), mediante el Programa Fondo para el Apoyo a Proyectos Productivos en Núcleos Agrarios (FAPPA) 2016. Se obtuvo el folio de acuse de registro: FP-CHIH-16-01012-132287.

\section{CONCLUSIONES}

Se elaboró el proyecto de inversión denominado "Diseño de una granja productora de trucha arcoiris (Oncorhynchus Mykiss) en estanques en el municipio de Guachochi, Chihuahua, con el fin de aprovechar la oportunidad de negocio que existe debido a las condiciones del clima y calidad del agua adecuadas para el cultivo.

El proyecto es técnicamente, mercadológicamente, económicamente, financieramente y ambientalmente viable. Se sometió a la convocatoria de la Secretaria de Agricultura, Ganadería, Desarrollo Rural, Pesca y Alimentación (SAGARPA), mediante el Programa Fondo para el Apoyo a Proyectos Productivos en Núcleos Agrarios (FAPPA) 2016, obteniéndose el folio de acuse de registro: FP-CHIH-1601012-132287.

En cuanto a los impactos ambientales se reducirá el impacto del consumo de energía eléctrica $(35 \%)$ mediante el uso de paneles solares. En cuanto al uso de las aguas de desecho se le dará el uso como agua para riego de cultivos a través de utilizar un sistema de filtro. El proyecto no modificará la dinámica natural de ningún cuerpo de agua, ya que solamente se utilizara para el llenado de los estanques utilizando el agua proveniente de un manantial. Además, el suelo no se verá afectado por la instalación de la granja, ya que no se harán excavaciones profundas, ni se expondrá a materiales corrosivos que pudieran afectarlo y el agua utilizada en los estanques será utilizada de manera racional evitando se tire y provoque erosión.

La acuicultura es una técnica de producción de peces que promueve el desarrollo regional sustentable al utilizar recursos naturales como el agua y el clima, que son ideales para la trucha arcoiris, sin embargo, hace falta información y asesoría técnica para fomentar la elaboración de proyectos en la región bajo estudio. Este proyecto servirá como empresa piloto para fomentar con los pobladores de la región, proyectos acuícolas rentables y ambientalmente comprometidos.

\section{LITERATURA CITADA}

Diario Oficial, (segunda sección) Secretaría de Agricultura, Ganadería, Pesca, Desarrollo Rural, FAO (2012) La formulación de proyectos de acuicultura (ftp://ftp.fao.org/fi/CDrom/FAO training/F AO training/general/x6709s/x6709s 16.htm Fecha de consulta 31 octubre 2015. Gobierno del Estado de Chihuahua página oficial http://www.chihuahua.gob.mx/atach2/Princi pal/uploads/diag(2).pdf Fecha de consulta 18 octubre 2015.

Hernández, A., Abraham, H. (1998). Formulación y evaluación de proyectos de inversión. 1 edición. Ediciones contables, Administrativas y Fiscales S.A de C.V. México, DF. PP. 28.

Huet, M., Benito Martinez, F.J., \& Timmermans, J. A. (1998). Tratado de piscicultura. $4^{a}$ edición. S.A. Mundi-Prensa Libros. Madrid, España. PP. 5-25.

INEGI (2015) México en cifras. Información nacional, por entidad federativa y municipios. Instituto Nacional de 
Estadística y Geografía. Av. Héroe de Nacozari Sur Núm. 2301 Fracc. Jardines del Parque C.P. 20276 Aguascalientes, Ags.México. (Disponible en línea en http://www3.inegi.org.mx/sistemas/mexicoc ifras/default.aspx?e=8 Fecha de consulta 30 de mayo 2015.

Jover, M., S, Martínez., A., Tomas., \& Pérez L. (2003). Propuesta metodológica para el diseño de instalaciones piscícolas. Revista AquaTic n¹9., Grupo de Investigación en Recursos Acuícolas, Dpto. Ciencia Animal, Universidad Politécnica de Valencia. pp. 1726. (Disponible en línea en http://www.revistaaquatic.com/aquatic/art.as $\mathrm{p} ? \mathrm{t}=\mathrm{p} \& \mathrm{c}=168)$. Fecha de consulta $30 \mathrm{de}$ mayo de 2015.

Municipio de Guachochi página oficial. Conoce Guachochi. (Disponible en línea en http://www.guachochi.gob.mx/) Fecha de consulta 10 de febrero 2016.

Pesca y Alimentación, 2012. ACUERDO mediante el cual se aprueba la actualización de la Carta Nacional Acuícola. (Disponible en línea en

http://www.inapesca.gob.mx/portal/docume ntos/publicaciones/2011/06062012\%20SAG ARPA.pdf). Fecha de consulta 16 de marzo de 2015.

Solís, MW (2008) Plan municipal de desarrollo Guachochi, ayuntamiento 20072010 (Disponible en línea en http://www.guachochi.gob.mx/atach2/guach ochi/canales/Adjuntos/CN_1098CC_1762/P LAN\%20DE\%20GUACHOCHI.pdf Fecha de consulta 10 de febrero 2016.

Woynarovich, A., Hoitsy, G. y MothPoulsen, T. (2011). Small-scale rainbow trout farming. FAO Fisheries and aquaculture technical paper No. 561. Roma, FAO. PP. 92.

\section{FIGURAS}

\section{Análisis de la fuente de agua.}

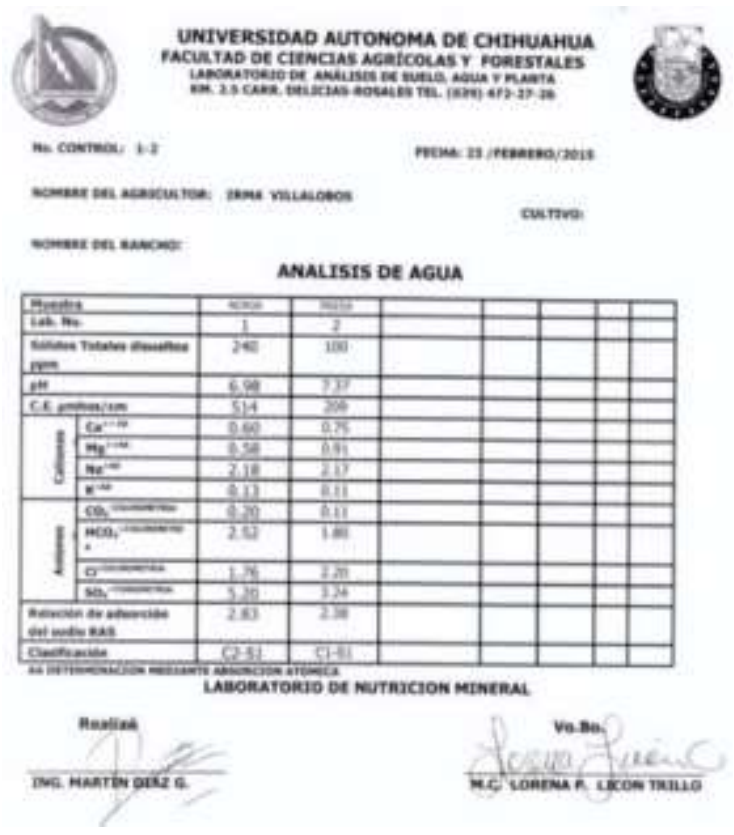

2. Análisis del agua. Indicadores.

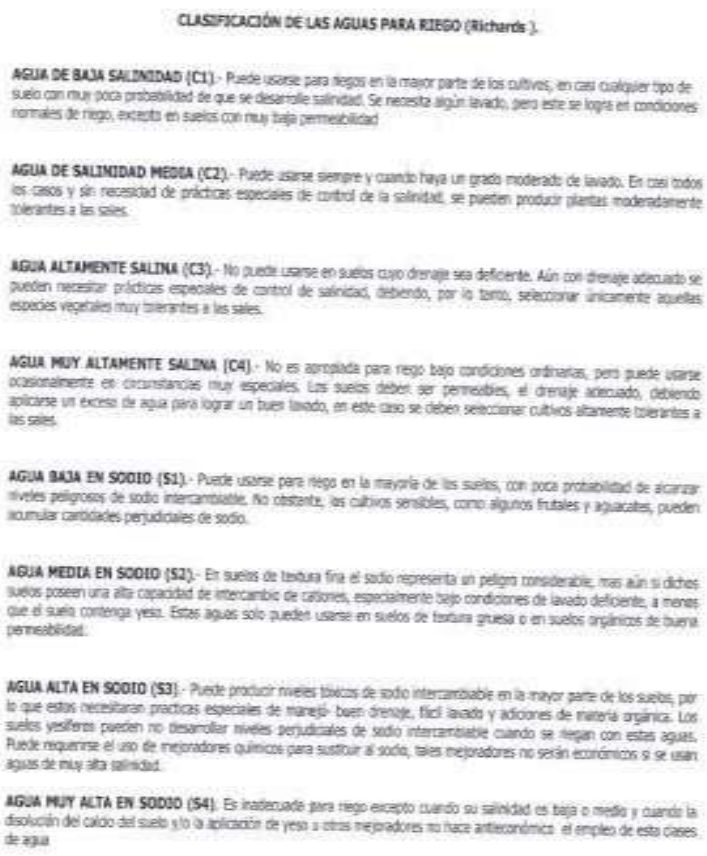


3. Mano de obra.

\begin{tabular}{|c|c|c|c|c|}
\hline & MANO DE OBRA & & & AÑO \\
\hline $\begin{array}{l}\text { Administrador, } \\
\text { presidente. } \\
\text { Gil Díaz Acosta }\end{array}$ & $\begin{array}{l}\text { Administrar, supervisar los trabajos } \quad \text { de construcción y } \\
\text { ayudar en lo que sea necesario }\end{array}$ & 48 & $\$ 100.00$ & $\$ 4,800.00$ \\
\hline $\begin{array}{l}\text { Cuidado y } \\
\text { alimentación de } \\
\text { los peces }\end{array}$ & $\begin{array}{l}\text { Se necesitara una persona todos los días del año para } \\
\text { cuidado de los peces, alimentación labores generales de } \\
\text { cuidado de los estanques }\end{array}$ & 260 & $\$ 100.00$ & $\$ 26,000.00$ \\
\hline $\begin{array}{l}\text { Mantenimiento } \\
\quad \text { de los } \\
\text { estanques y } \\
\text { compra de } \\
\text { insumos } \\
\text { Soledad Acosta } \\
\text { Lerma }\end{array}$ & $\begin{array}{l}\text { Mantenimiento de los estanques y encargado de la venta de } \\
\text { los peces, entregas, etc. }\end{array}$ & 4 & $\$ 120.00$ & $\$ 480.00$ \\
\hline \multicolumn{4}{|c|}{ TOTAL } & $\$ 31,280.00$ \\
\hline
\end{tabular}

\section{Costo y alimentación de alevines.}

\begin{tabular}{|c|c|c|c|c|c|c|c|}
\hline \multicolumn{8}{|c|}{ COSTOS DE ALEVINES, ALIMENTACION Y ENFERMEDADES } \\
\hline & & Caracteristicas biologicas & & Cap. Alevines & No de estanques & Total & $\begin{array}{c}2 \text { Cosechas al } \\
\text { año }\end{array}$ \\
\hline \multirow{6}{*}{ Etapa } & Alevines & $\begin{array}{l}\text { Esta etapa dura en } \\
\text { promedio } 15 \text { dias } \\
\text { mortalidad alevines } 8 \% \\
\text { tamaño de } 4 \text { a } 6 \mathrm{~cm} \\
\text { peso de } 0.7 \text { a } 3 \mathrm{grs}\end{array}$ & $\begin{array}{l}\text { Estanque de } 30 \mathrm{~m} \text { largo, } \\
3 \mathrm{~m} \text { de ancho, } 1 \mathrm{~m} \text { de } \\
\text { profundidad }=90 \mathrm{~m} 3 \mathrm{Capac} \\
\text { idad }=40 \text { Alevines por } \mathrm{m} 3\end{array}$ & 3600 & 4 & 14400 & \\
\hline & Juveniles & $\begin{array}{l}\text { Esta etapa dura en } \\
\text { promedio } 90 \text { dias } \\
\text { mortalidad juveniles } 4 \% \\
\text { tamaño de } 10 \text { a } 15 \mathrm{~cm} \\
\text { peso de } 11 \text { a } 40 \mathrm{grs}\end{array}$ & & 3312 & 4 & 13248 & \\
\hline & Talla comercial & $\begin{array}{l}\text { Esta etapa dura } 105 \text { dias } \\
\text { mortalidad talla } \\
\text { comercial } 3 \% \\
\text { tamaño de } 21 \text { a } 25 \mathrm{~cm} \\
\text { peso } 250 \mathrm{grs}\end{array}$ & & 3212.64 & 4 & 12850.56 & \\
\hline & Vental & kgrs & & 803.16 & 4 & 3212.64 & 6425.28 \\
\hline & $\begin{array}{l}\text { Precio de } \\
\text { venta/kgr }\end{array}$ & $\$ 100$ & & $\$ 8,031.60$ & 4 & $\$ 32,126$ & $\$ 642,500.00$ \\
\hline & $\begin{array}{c}\text { Adquisicion de } \\
\text { alevines, costo } \\
\text { unitario }\end{array}$ & 1.7 & & 14400 & 2 & 28800 & 48960 \\
\hline
\end{tabular}

Revista Científica Biológico Agropecuaria Tuxpan 5 (1) 


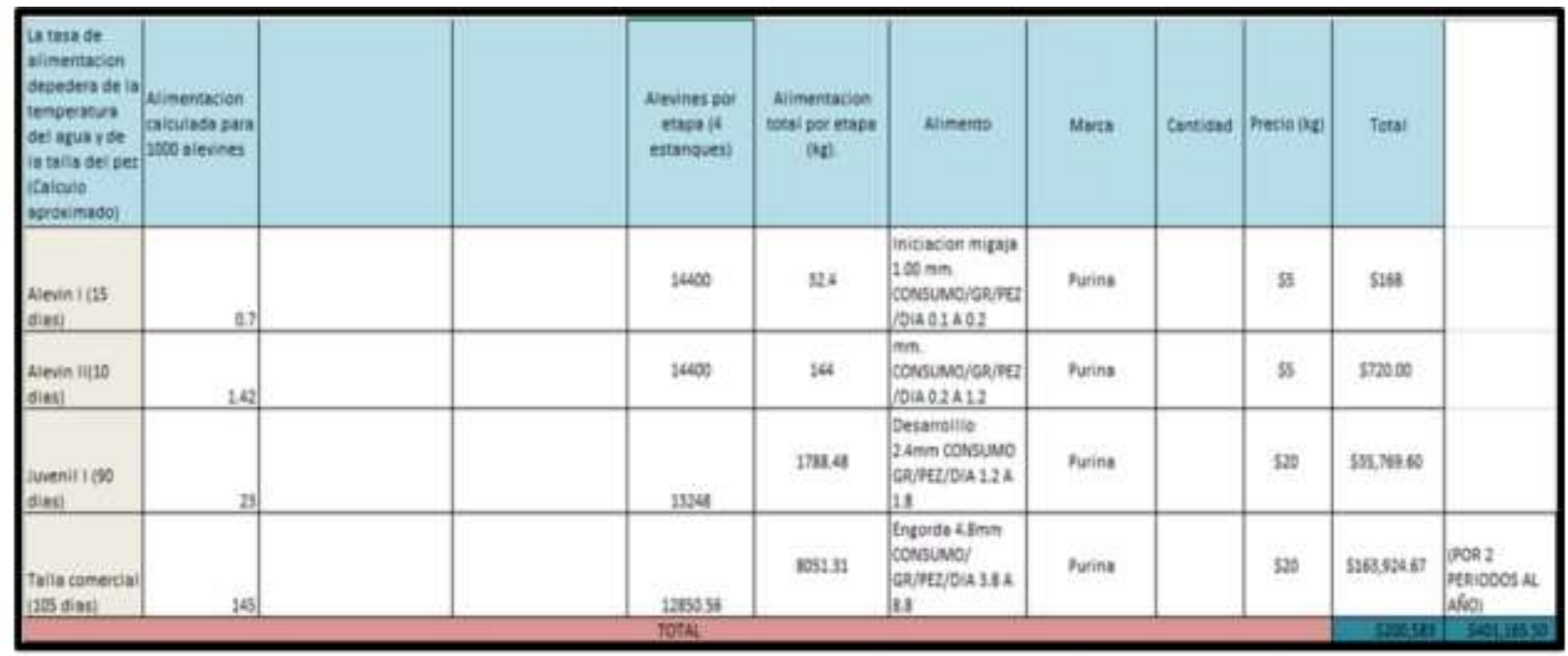

\begin{tabular}{|c|c|c|c|c|c|c|c|}
\hline \multirow{5}{*}{$\begin{array}{l}\text { Manejo de } \\
\text { enfermedsdes }\end{array}$} & Entermediad & Medicamento & Unidad de medida & Mares & frecio unitario & Cantidad & Total \\
\hline & $\begin{array}{l}\text { Prevencion de } \\
\text { enfermedades } \\
\text {, parasitos. }\end{array}$ & $\begin{array}{l}\text { Sal blanca para zanado: } \\
\text { Barlos con solución de } \\
\text { sal común al } 1 \mathrm{s,}, 3 \cdot 4 \\
\text { tratamientos a intervalos } \\
\text { de } 3 \text { dias. }\end{array}$ & Bulto & & 570.00 & 1 & 570.00 \\
\hline & Eacterias. & $\begin{array}{c}\text { Terramicina } \\
\text { (Oxitetraciclina) Polvo } \\
\text { soluble formula animal. } \\
3 \mathrm{~g} / \mathrm{cada} 45 \mathrm{~kg} \text { de pez }\end{array}$ & Bolsa 200 gramos & Pfizer & $\$ 92.00$ & 1 & $\$ 9200$ \\
\hline & $\begin{array}{c}\text { Desinfectante } \\
\text { para } \\
\text { estanques }\end{array}$ & Virkon 108 & Pieza & Baver & 54500 & 1 & $\$ 45.00$ \\
\hline & \multicolumn{6}{|c|}{ TOTAL } & $\$ 20700$ \\
\hline
\end{tabular}


Copyright (c) 2017 Irma Ari sbel Villal obos Gracia, José E duardo Magaña Magaña,

Victor Hugo Villarreal Ramirez, JoséE steban Hernández Salas, Juan Javier Núñez L ópez

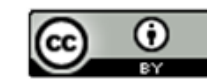

Este tex to está protegido por una licencia licencia CreativeCommons 4.0.

Usted es libre para Compartir —copiar y redistribuir el material en cualquier medio o form ato-y Adaptar el documento —remezclar, transformar y crear a partir del material- para cualquier propósito, incluso para fines comerciales, siempre que cumpla la condición de:

Atribución: Usted debe dar crédito a la obra original de manera adecuada, proporcionar un enlace a la licencia, e in dicar si se han realizado cam bios. Puede hacerlo en cualquier form a razonable, pero no de form a tal que sugiera que tiene el apoyo del licenciante o lo recibe por el uso que hace de la obra.

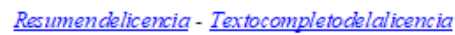

